\section{CONTRIBUIÇÃO AO ESTUDO DA IRRIGA- ÇAO ARTERIAL DO NÓ SINOATRIAL NO JUMENTO NORDESTINO}

ANTONIO FERNANDES FILHO

Professor Adjunto

Faculdade de Medicina Veterinária e

Zootecnia da USP

VICENTE BORELLI

Professor Titular

Faculdade de Medicina Veterinária e

Zootecnia da USP

IRVENIA LUIZA DE SANTIS PRADA

Professora Adjunta

Faculdade de Medicina Veterinária e

Zootecnia da USP

\section{EUCLIDES FEITOSA FILHO}

Professor Adjunto

Departamento de Morfologia e Fisiologia Animal

Universidade Federal Rural de Pemambuco

FERNANDES FILHO, A.; BORELLI, V.; PRADA, I.L.S.; FEITOSA FILHO, E. Contribuição ao estudo da irrigação arterial do nó sinoatrial no jumento nordestino. Rev.Fac.Med.vet. Zootec.Univ.S.Paulo, 22(1): 7-14, 1985.

RESUMO: A irrigação arterial do nó sinoatrial foi estudada em 30 corações de jumentos nordestinos, adultos, 15 machos e 15 fêmeas. Observou-se que o nó sinoatrial apresenta-se nutrido exclusivamente pelo ramus proximalis atrii sinistri -21 vezes $(70,0 \% \pm 8,3)$; pelos rami proximalis atrii sinistri e intermedius atrii dextri -4 vezes $(13,3 \% \pm 6,2)$; pelos rami proximaiis atrii sinistri e distalis atrii dextri -3 vezes $(10,0 \% \pm 5,4)$ e pelo ramus proximalis atrii dextri - 2 vezes $(6,7 \% \pm 4,5)$. Nāo foram identificadas anastomoses entre vasos implicados na irrigação arterial do nó sinoatrial. Não existem influências, relativamente ao sexo, quando se consideraram as diferentes modalidades de suprimento sangüineo do nó sinotrial.

UNITERMOS: Anatomia, equinos ${ }^{+}$; Coraçāo $^{+}$; Coronárias ${ }^{+}$; Nó sinoatrial; Jumento nordestino

\section{INTRODUÇÃO E LITERATURA}

O comportamento dos vasos responsáveis pela irrigação do nó sinoatrial em jumentos nordestinos, representa náo só a continuidade de nossas pesquisas sobre o assunto mas, também, a preocupação de melhor conhecer este animal, de incontestável importância, particularmente na Região Nordeste do Brasil.

BORELLI et aliii (1982), em outra oportunidade, informam estar o referido tecido nodal, nestes espécimes, localizado na face esquerda da junção da veia cava cranial com o átrio direito, ângulo diedro cavo-atrial e terço superior do sulco terminal.

De outra parte, se considerarmos a vascularização arterial desta estrutura, nos eqüídeos, PACE $^{9}$ (1925) em estudo anatomoradiológico sobre Aa. coronárias do cavalo, esclarece que em um coraçăo o nó do seio é nutrido por colateral da $\mathrm{A}$. coronária direita e em outro por colateral da $\mathrm{A}$. coronária esquerda.

Já, ERHART ${ }^{3}$ (1936), baseado na dissecção de 5 coraçōes de cavalo, registra que o citado sistema depende quase que exclusivamente de colateral da $\mathbf{A}$. coronária esquerda, isto é, do ramo auricular anterior esquerdo, lembrando que a A. coronária direita fornece o ramo auricular posterior direito, para a porção baixa do sulco terminal.

FERNANDES FILHO 4 (1969), examinando 50 coraçōes de cavalos da raça Puro Sangue Inglês, adultos, 26 machos e 24 fêmeas, com idade variando entre 2 e 7 anos, relata que o nó sinoatrial mostra-se servido, 26 vezes $(52,0 \% \pm$ $7,1)$ por colaterais das Aa. coronaria dextra e coronaria sinistra, isto é, pelo ramus distalis atrii dextri associado ao ramus proximalis atrii sinistri $(42,0 \% \pm 7,0)$ ou combinadamente pelos rami intermedius atrii dextri e proximalis atrii sinistri $(10,0 \% \pm 4,2)$. Descreve ainda o autor, que o referido nó é nutrido 24 vezes $(48,0 \% \pm 7,1)$ pelo ramus distalis atrii dextri, exclusivamente $(44,0 \% \pm 7,0)$ ou por este vaso associado ao ramus intermedius atrii dextri $(4,0 \%$ $\pm 2,8$ ), orindos da A. coronaria dextra.

Ainda, FERNANDES FILHO et alii ${ }^{7}$ (1971), estudando 50 coraçōes de cavalos, sem raça definida, adultos, 25 machos e 25 fêmeas, registram que a irrigação arterial do nó sinoatrial depende, com maior frequência 48 vezes $(96,0 \% \pm 2,8)$, de colaterais oriundos de ambas as coronárias, isto é, do ramus proximalis atrii sinistri associado ao ramus distalis atrii dextri, 29 vezes $(58,0 \% \pm 7,0)$; ao ramus intermedius atrii dextri, 18 vezes $(36,0 \% \pm 6,8)$ e aos rami intermedius atrii dextri e distalis atrii dextri -1 vez $(2,0 \% \pm 2,0)$, ficando raramente este tecido nodal na dependência exclusiva de colaterais da $\mathrm{A}$. coronária dextra, isto é, apenas do ramus distalis atrii dextri, 1 vez $(2,0 \% \pm$ $2,0)$ ou deste associado ao ramus proximalis atrii dextri, 1 vez $(2,0 \% \pm 2,0)$.

De outra parte, FERNANDES FILHO et alii ${ }^{5}$ (1972), analisando 50 coraçð̃es de muares, adultos, 39 machos e 11 fêmeas, esclarece que o nó sinoatrial apresenta-se irrigado 
-41 vezes $(82,0 \% \pm 5,5)$ por colateral da $\mathrm{A}$. coronária sinistra, isto é, pelo ramus proximalis atrii sinistri, 8 vezes $(16,0 \% \pm 5,2)$ por colaterais de ambas as coronárias, mais exatamente pelo ramus proximalis atrii sinistri aliado ao ramus distalis atrii dextri, 5 vezes ou ao ramus intermedius atrii dextri, 3 vezes e 1 vez $(2,0 \% \pm 2,0)$ apenas por colateral da A. coronaria dextra, isto é, pelo ramus proximalis atrii dextri.

Finalmente, FERNANDES FILHO et alii6 (1972), pesquisando em 45 coraçøes de asininos da raça Pêga, adultos, 24 fêmeas e 21 machos, a irrigaçao do nó sinoatrial, evidenciam que esta estrutura acha-se nutrida 40 vezes $(88,9 \% \pm 4,6)$ exclusivamente por colateral da A. coronaria sinistra, vale dizer, pelo ramus proximalis atrii sinistri e 5 vezes $(11,1 \% \pm 4,6)$ por colateral da $\mathbf{A}$. coronaria dextra, isto é, pelo ramus proximalis atrii dextri.

Cabe ainda elucidar que, dentre os tratadistas, apenas BRUNI \& ZIMMERL ${ }^{2}$ (1951) assinalam genérica e sumariamente que a artéria nodal deriva da $A$. coronaria dextra e corre ao longo do sulco terminal.

\section{MATERIAL E MÉTODO}

Examinamos neste trabalho 30 coraçoes de jumentos nordestinos, adultos, 15 machos e 15 fêmeas, oriundos de diversos estados do Nordeste e abatidos no Matadouro Frigorifico Industrial S/A., em Belo Jardim, Pernambuco.

Depois de isolado o órgão, esvaziados e lavados os ventrículos e átrios, injetamos separadamente, as artérias coronárias com Neoprene latex " 450 ", corado de verde e, a seguir, procedemos à dissecção destes vasos, após terem sido os coraçðes fixados em solução aquosa de formol a $10,0 \%$.

De todas as preparaçoes efetuamos desenhos esquemáticos para ulterior análise, sendo que para a descrição dos resultados adotamos a nomenclatura empregada por HOFFMAN $^{8}$ (1960).

\section{RESULTADOS}

O território ocupado pelo nó sinoatrial é nutrido mais comumente, 21 vezes $(70,0 \% \pm 8,3-$ Obs. $3 \mathrm{~m}, 4 \mathrm{~m}, 6 \mathrm{~m}, 7 \mathrm{~m}$, $8 \mathrm{~m}, 10 \mathrm{~m}, 11 \mathrm{~m}, 12 \mathrm{~m}, 14 \mathrm{~m}, 15 \mathrm{~m}, 16 \mathrm{f}, 17 \mathrm{f}, 18 \mathrm{f}, 20 \mathrm{f}, 21 \mathrm{f}$, 25f, 26f, 27f, 28f, 29f, 30f - Fig. 1), apenas pelo ramus proximalis atrii sinistri, colateral do ramus circunflexus da A. coronaria sinistra. Este vaso, após contornar a parede côncava dos átrios, percorre, como tronco, a desembocadura da veia cava cranial, na sua face auricular para alcançar o ângulo diedro cavo-atrial e o sulco terminal, no mínimo no seu terço superior.

No traje to descrito, o ramus proximalis atrii sinistri cede, ainda, contribuiç̧es às faces auricular $e$ atrial do átrio esquerdc, à regiáo da desembocadura das veias pulmonares, ao septo interatrial, à secção final da veia cava cranial e a face auricular do átrio direito.

Em algumas preparaçðes, 7 vezes $(23,3 \% \pm 7,7-$
Obs. $1 \mathrm{~m}, 5 \mathrm{~m}, 9 \mathrm{~m}, 19 \mathrm{f}, 22 \mathrm{f}, 23 \mathrm{f}, 24 \mathrm{f}$ - Fig 2, 3), o ramus proximalis atrii sinistri, oriundo do ramus circunflexus da A. coronaria sinistra apresenta comportamento semelhante ao já descrito, quando sozinho se incumbe da nutrição da área tomada pelo nó sinoatrial, todavia, não alcança o terço superior do sulco terminal, que nestes casos é irrigado por colaterais do ramus circumflexus da $\mathbf{A}$. coronaria dextra, mais exatamente pelos rami intermedius atrii dextri, 4 vezes $(13,3 \% \pm 6,2-$ Obs. $1 \mathrm{~m}, 9 \mathrm{~m}, 23 \mathrm{f}, 24 \mathrm{f}-$ Fig. 2$)$ e o distalis atrii dextri, 3 vezes $(10,0 \% \pm 5,4$ - Obs. $5 \mathrm{~m}, 19 \mathrm{f}, 22 \mathrm{f}$, - Fig. 3). Nestes coraçoes, o ramus intermedius atrii dextri que nasce entre as emergências dos rami marginis convexi e distalis ventriculi dextri, 2 vezes (Obs. $1 \mathrm{~m}, 24 \mathrm{f}$ ) ou entre a saída dos rami proximalis ventriculi dextri e marginis convexi, 2 vezes (Obs. $9 \mathrm{~m}, 23 \mathrm{f}$ - Fig. 2) e o ramus distalis atrii dextri que nasce entre as origens dos rami marginis convexi e distalis ventriculi dextri, 3 vezes (Obs. $5 \mathrm{~m}, 19 \mathrm{f}$, $22 \mathrm{f}$ - Fig. 3) ganham a face atrial do átrio direito para alcançarem, como tronco, o terço superior do sulco terminal, cedendo ainda colaterais à face atrial do átrico direito, à seç̧a terminal da veia cava cranial e o último deles, também à secção final da veia cava caudal.

Em poucas preparaçסes, 2 vezes $(6,7 \% \pm 4,5-$ Obs. $2 \mathrm{~m}, 13 \mathrm{~m}$ - Fig. 4 ) cabe exclusivamente ao ramus proximalis atrii dextri, a irrigação da área ocupada pelo nó sinoatrial. Este vaso que se origina do ramus circumflexus da $\mathbf{A}$. coronaria dextra, antes da emergência da A. adiposa, ganha a face auricular do átrio direito e com trajeto ascendente alcança, como tronco, a desembocadura da veia cava cranial para ganhar o ângulo diedro cavo-atrial e percorrer o sulco terminal, em toda sua extensăo.

Nestes casos, o ramus proximalis atrii dextri fornece, ainda, colaterais à face auricular do átrio e auricula direitos, à face auricular do átrio esquerdo, à secçáo final das veias pulmonares e da cava caudal e à face atrial do átrio direito.

\section{COMENTÁRIOS}

O nó sinoatrial, que foi identificado nos jumentos nordestinos ocupando a face esquerda da junção cavo-atrial, ângulo diedro cavo-atrial e terço superior do sulco terminal, recebe mais comumente, 21 vezes $(70,0 \% \pm 8,3)$ apenas o ramus proximalis atrii sinistri, este e os rami intermedius atrii dextri, 4 vezes $(13,3 \% \pm 6,2)$ ou o distalis atrii dextri, 3 vezes $(10,0 \% \pm 5,4)$ ou ainda somente o ramus proximalis atrii dextri, 2 vezes $(6,7 \% \pm 4,5)$.

Se considerarmos, inicialmente, os trabalhos de FERNANDES FILHO ${ }^{4}$ e FERNANDES FILHO $5,6,7$, os resultados ora obtidos muito se aproximam dos encontrados em muares e asininos, que também apresentam, mais comumente, apenas o ramus proximalis atrii sinistri incumbindo-se da nutrição deste tecido nodal, isto é 41 vezes $(82,4$. $\pm 5,4)$ no primeiro caso e 40 vezes $(88,9 \% \pm 4,6)$ no segundo e ainda raramente nestes animais, o ramus proximalis atrii dextri desempenha sozinho esta função, fato observado 
apenas 1 vez $(2,0 \% \pm 2,0)$ e 5 vezes $(11,1 \% \pm 4,6)$, respectivamente.

Já, a associação dos ramus proximalis atrii sinistri com os rami intermedius atrii dextri ou com o distalis atrii dextri, vasos que ocasionalmente são encontrados nutrindo o nó sinoatrial no jumento nordestino, bem como nos muares, isto é, 3 vezes $(6,0 \% \pm 3,4)$ e 5 vezes $(10,0 \% \pm$ $4,2)$, surgem como principais responsáveis por esta tarefa nos cavalos sem raça definida, isto é, por ordem, 29 vezes $(58,0 \% \pm 7,0)$ e 18 vezes $(36,0 \% \pm 6,8)$, sendo que também o cavalo P.S.I. mostra com significativa frequência, isto é, 21 vezes $(42,0 \% \pm 7,0)$, a participação conjunta dos rami proximalis atrii sinistri e distalis atrii dextri.

De outra parte, não foi identificada nos jumentos nordestinos, a presença concomitante de três vasos no território ocupado pelo nó sinoatrial, vale dizer os rami proximalis atrii sinistri, intermedius atrii dextri e distalis atrii dextri, observados, em poucas preparaçסes apenas no cavalo P.S.I., 5 vezes $(10,0 \% \pm 4,2)$ e no cavalo sem raça definida, 1 vez $(2,0 \% \pm 2,0)$, ocorrendo também, somente nestes espécimes, a participação isolada do ramus distalis atrii dextri, isto é, 27 vezes $(44,0 \% \pm 7,0)$ e 1 vez $(2,0 \% \pm 2,0)$, respectivamente, bem como a combinação deste vaso com o ramus intermedius atrii dextri, 2 vezes $(4,0 \% \pm 2,8)$ no cavalo P.S.I. ou com o ramus proximalis atrii dextri, 1 vez $(2,0 \% \pm 2,0)$ no cavalo sem raça definida.

As diferenças ora assinaladas ocorrem, a nosso ver em função das variaçбes raciais, todavia, devemos lembrar que a diversidade dos arranjos vasculares dependem, também, da topografia do nó sinoatrial que nos jumentos nordestinos, muares e asininos,localiza-se no terço superior do sulco terminal, ângulo diedro cavo-atrial e face esquerda da junção cavo-atrial e nos cavalos P.S.I. e sem raça definida, em toda extensão do sulco terminal e no referido ângulo. Este fato talvez possa explicar a similitude das disposiçōes vasculares nestes dois grupos de animais, bem como a maior participaçđo dos rami intermedius atrii dextri e distalis atrii dextri na nutriçáo do nó sinoatrial dos cavalos P.S.I. e sem raça definida.

Ainda, cabe lembrar que anastomoses arteriais de que participam os vasos responsáveis pela irrigação do nó sinoatrial, de pequena ocorrência nos equídeos, nđo foram assinaladas nos jumentos nordestinos.

Finalmente, devemos esclarecer que consideramos genéricas e imprecisas as informaçס̃es de PACE 22 e BRUNI \& ZIMMERL ${ }^{2}$, enquanto que as observaçōes de ERHART ${ }^{3}$, realizadas em cavalos, coincidem apenas parcialmente com os nossos achados, em jumentos nordestinos.

\section{CONCLUSŌES}

O estudo ora realizado em 30 coraçōes de jumentos nordestinos, 15 machos e 15 fêmeas, adultos, permite-nos as seguintes conclusoes:

1) a região ocupada pelo nó sinoatrial depende nestes animais, mais frequentemente 21 vezes $(70,0 \% \pm 8,3)$ apenas de colateral oriundo do ramus circumflexus da A. coronaria sinistra, isto é, do ramus proximalis atrii sinistri;

2) o tecido nodal recebe em alguns coraçðes, 7 vezes $(23,3 \% \pm 7,7)$ colaterais procedentes dos rami circumflexus, de ambas as coronárias, mais exatamente o ramus proximalis atrii sinistri associado ao ramus intermedius atrii dextri, 4 vezes $(13,3 \% \pm 6,2)$ ou ao ramus distalis atrii dextri, 3 vezes $(10,0 \% \pm 5,4)$;

3) o nó sinoatrial mostra-se raramente, 2 vezes $(6,7 \% \pm$ $4,5)$ servido somente por colateral do ramus circumflexus da A. coronaria dextra, isto é pelo ramus proximalis atrii dextri;

4) nāo existem diferenças estatisticamente significantes ao nivel de $5 \%$, relativamente ao sexo, quando consideramos na irrigação da massa nodal a participação exclusiva de colaterais oriundos do ramus circumflexus da A. coronaria sinistra, ou da $\mathbf{A}$. coronaria dextra, bem como nos casos de participaçáo de colaterais dos rami circumflexus de ambas as coronárias.

FERNANDES FILHO, A.; BORELLI, V.; PRADA, I.L.S.; FEITOSA FILHO, E. Contribution of the study of the blood supply of the sinus node of donkeys. Rev.Fac.Med.vet.Zootec. Univ.S.Paulo, 22(1): 7-14,1985.

SUMMARY: The arterial blood supply of the sinus node has been studied in 30 donkeys: 15 males and 15 females. It was observed that the sinus node was nourished exclusively by the ramus proximalis atrii sinistri in $21(70.0 \% \pm 8.3)$ of the examined hearts; by the ramus proximalis atrii sinistri and ramus intermedius atrii dextri in $4(13.3 \% \pm 6.2)$ of the examined pieces; by the ramus proximalis atrii sinistri and ramus distalis atrii dextri in $3(10.0 \% \pm 5.4)$ of the examined organs; by the ramus proximalis atrii dextri in $2(6.7 \% \pm$ 4.5) of the examined cases. Furthermore, it was not evident the presence of anastomosis between the implied vessels in the arterial blood supply of the sinus node. Finally there was no sexual influence in the pattern of the blood supply to the sinus node.

UNITERMS: Anatomy of equines ${ }^{+}$; Heart $^{+}$; Coronary vessels ${ }^{+}$; Sinus node; Donkeys 


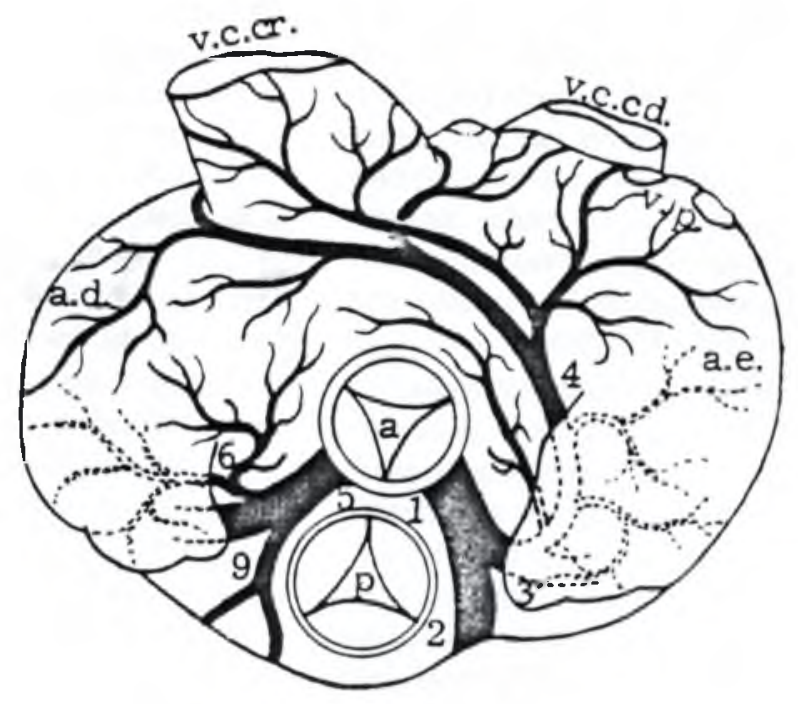

basis

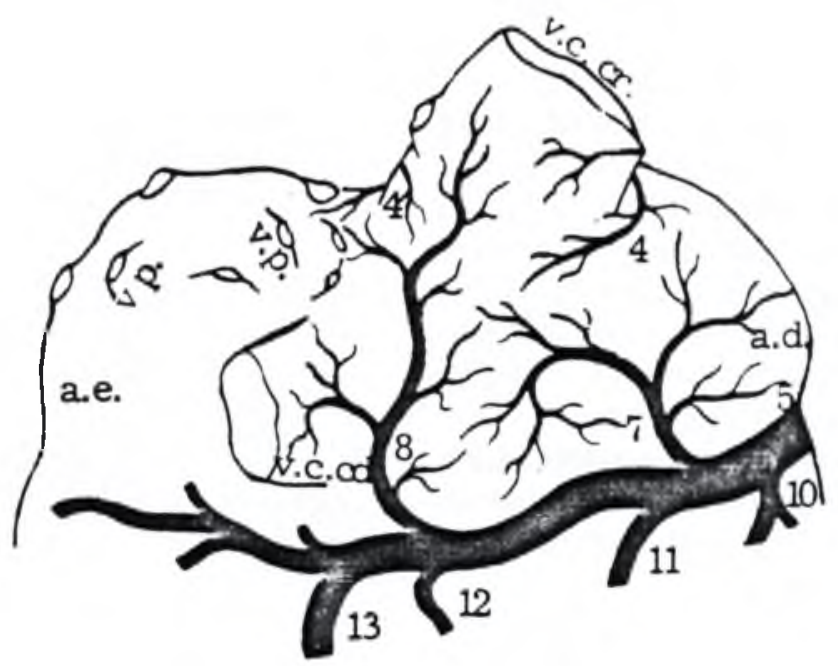

facies atrialis

FIGURA 1 - Obs. $12 \mathrm{~m}$ 


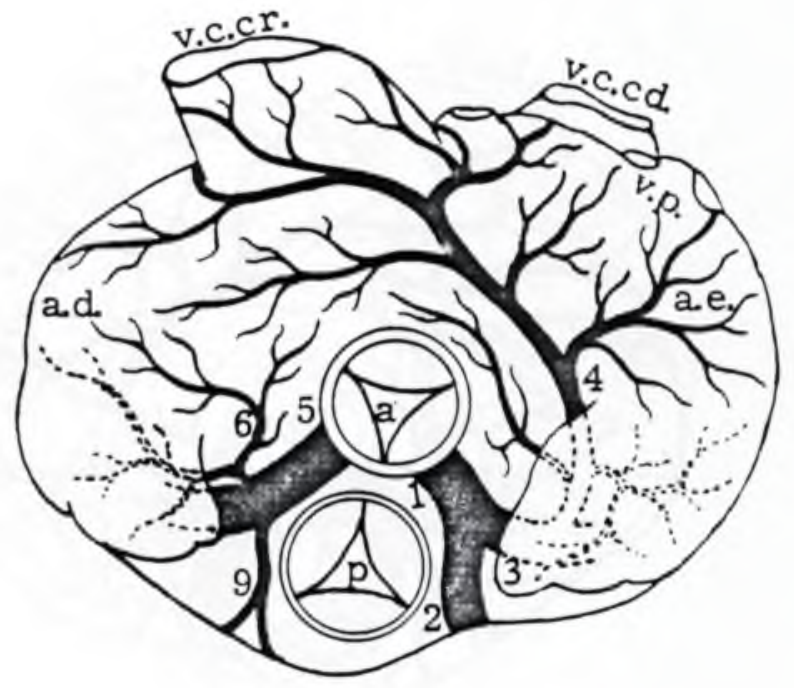

basis

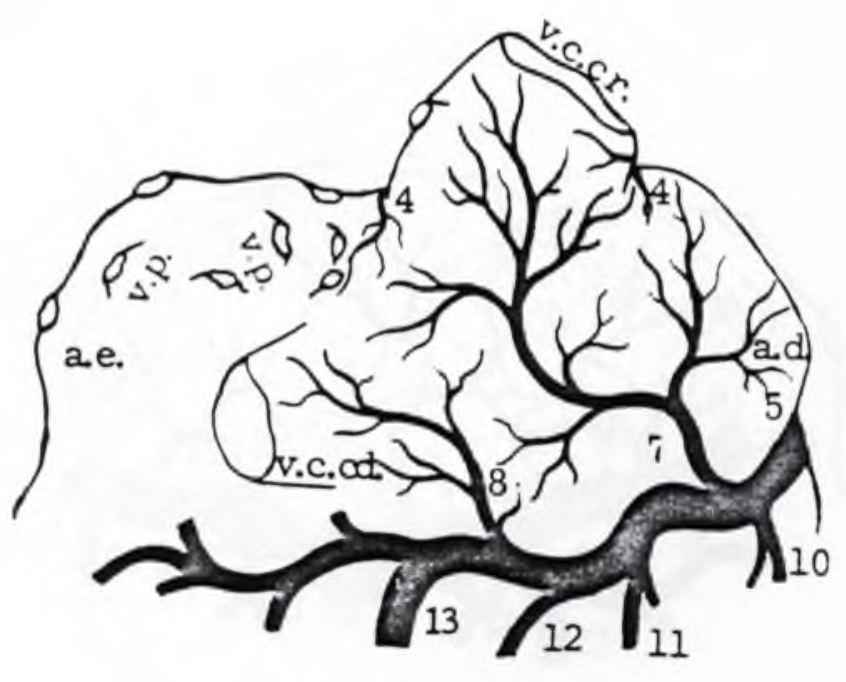

facies atrialis

FIGURA 2 - Obs. $9 \mathrm{~m}$ 


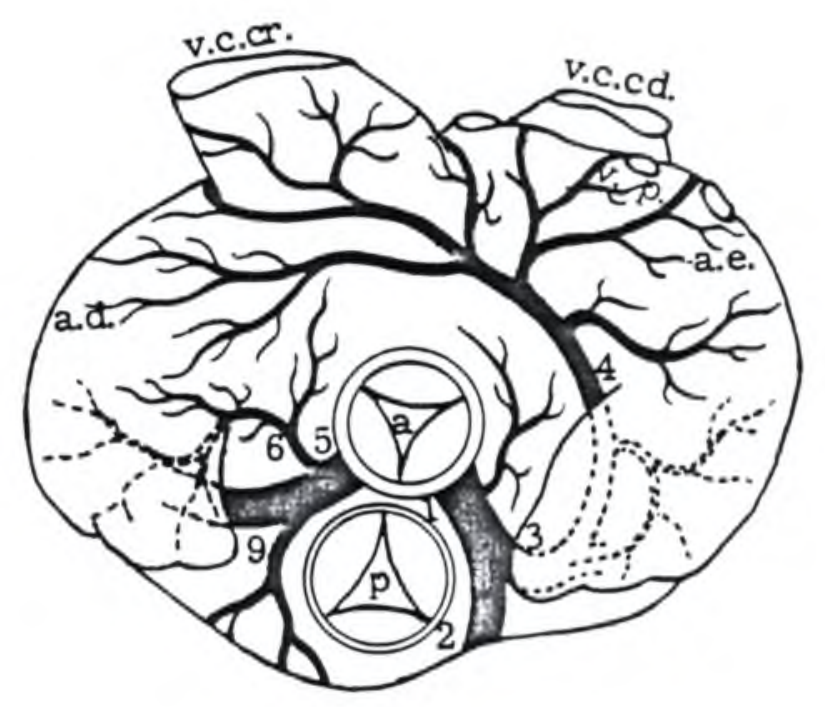

basis

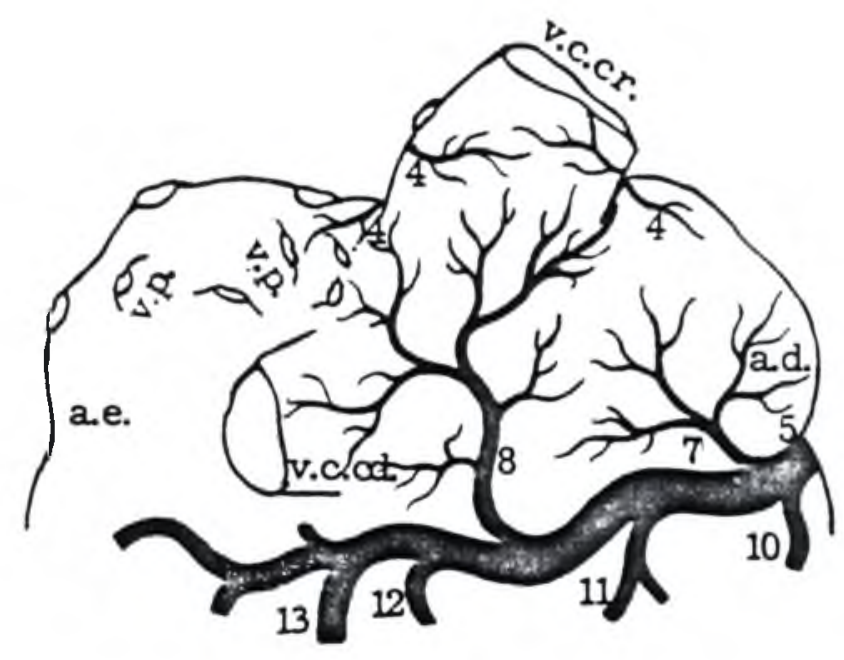

facies atrialis

FIGURA 3 - Obs. $19 f$ 


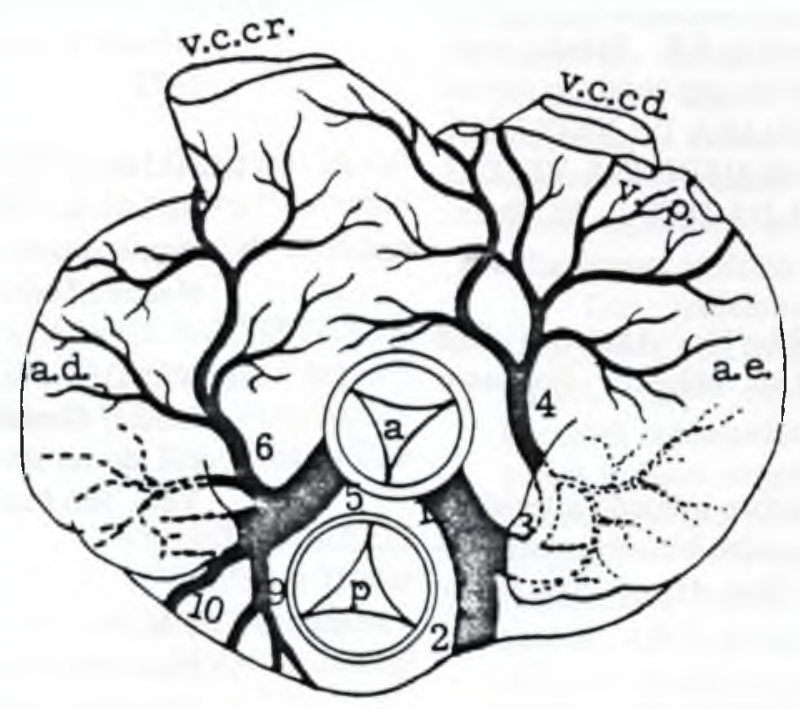

basis

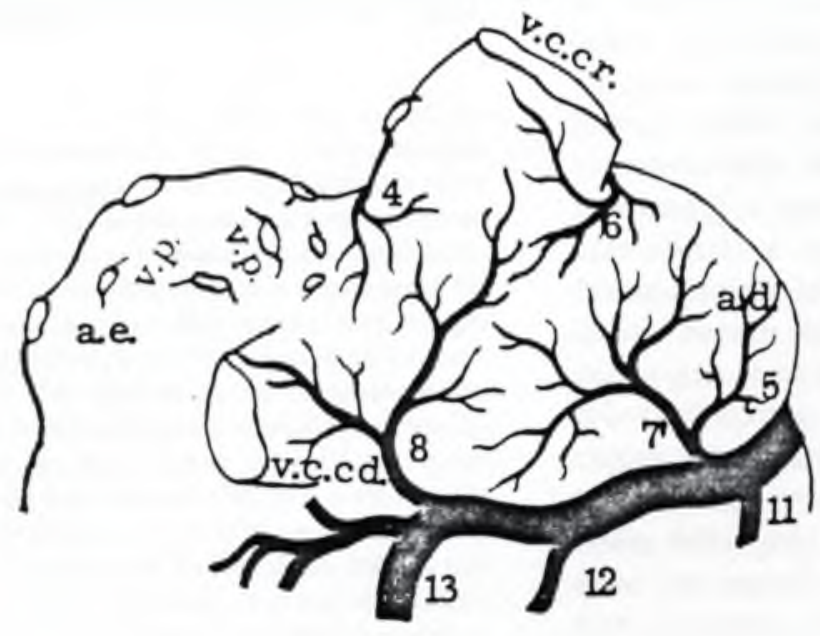

facies atrialis

FIGURA 4 - Obs. $2 \mathrm{~m}$ 


\section{REFERENCIAS BIBLIOGRĀFICAS}

1- BORELLI, V.; FERNANDES FILHO, A.; FEITOSA FILHO, E.; BOMBONATO, P. Estudo topográfico e histológico do nó sinu-atrial, no jumento nordestino. In: SEMANA DE VETERINÁRIA. FACULDADE DE MEDICINA VETERI. NÁRIA E ZOOTECNIA DA USP. 1., São Paulo, 1982. p.29.

2- BRUNI, A.C. \& ZIMMERL, U. Anatomia degli animali domestici. 2.ed. Milano, Francesco Vallardi, 1951.

3- ERHART, M.B. Pesquisas sobre irrigação arterial do sistema sinu-atrial do coração em alguns mamíferos domésticos. Rev. Biol. Hyg., São Paulo, $7(1): 1-14,1936$.

4- FERNANDES FILHO, A. Contribuiçăo ao estudo da irrigação arterial do nó sinu-atrial no cavalo Puro Sangue Inglês. Rev. Fac. Med. vet., São Paulo, 8:43-58, 1969.

5- FERNANDES FILHO, A.; BORELLI, V.D'ERRICO, A.A. Contribution a l'etude de lirrigation arterielle du noeud sinusal chez les mulets (Equus caballus x Equus asinus). Mammalia, 36:687-95, 1972.

6- FERNANDES FILHO, A.; BORELLI, V.; PEDUTI NETO, J. Contribuição ao estudo da irrigaçāo arterial do nó sinu-atrial em asininos. Rev.Fac. Med.vet Zootec.Univ S.Paulo, 9:5-12, 1972.

7- FERNANDES FILHO, A.; BORELLI, V.; PEREIRA J.G.L. Contribuiçăo ao estudo da irrigaçāo arte rial do nó sinu-atrial em eqüinos. Rev.Fac.Med Vet., São Paulo, 8:583-92, 1971.

8- HOFFMAN, V. Die Blutgefassversorgung des Pferdeherzens. Giessen, 1960. Inaugural. Dissertation-Fachbereich Veterinarmedizin und Tierzucht der Justus-Liebig-Universitat/

9- PACE, D. Saggio di uno studio anatomo radiografico sulle arterie coronarie nell'uomo e nei mammiferi. Arch. ital. Anat. Embriol., 22:247.82, 1925. 